\title{
Association Between Physical Performance and Cognitive Function in Chinese Community-Dwelling Older Adults: Serial Mediation of Malnutrition and Depression
}

\author{
Xinze Wu, ${ }^{1,2, *}$ \\ Guozhen Hou, ${ }^{1,2, *}$ \\ Peipei Han,' Xing Yu,' \\ Xiaoyu Chen,' Peiyu Song, ${ }^{3}$ \\ Yuanyuan Zhang, \\ Yinjiao Zhao, ${ }^{3}$ Fandi Xie, ${ }^{3}$ \\ Shumeng $\mathrm{Niu}^{3} \mathrm{HaO} \mathrm{Hu},{ }^{3}$ \\ Chengyi Sun,' Yuechen Zhao,' \\ Hongbing Wang, ${ }^{4}$ Qi Guo' \\ 'Department of Rehabilitation Medicine, \\ Shanghai University of Medicine and \\ Health Sciences, Shanghai, People's \\ Republic of China; ${ }^{2}$ College of Exercise \\ and Health Science, Tianjin University of \\ Sport, Tianjin, People's Republic of China; \\ ${ }^{3}$ Shanghai Jiangwan Hospital, Shanghai, \\ People's Republic of China; ${ }^{4}$ Department \\ of Rehabilitation, Shanghai Fourth \\ Rehabilitation Hospital, Shanghai, People's \\ Republic of China
}

*These authors contributed equally to this work

Correspondence: Hongbing Wang Department of Rehabilitation, Shanghai Fourth Rehabilitation Hospital, Shanghai, 20040, People's Republic of China Email 15301799929@I63.com

Qi Guo

College of Rehabilitation Medicine, Shanghai University of Medicine and Health Sciences, 279 Zhouzhu Highway, Pudong New Area, Shanghai, 201318,

People's Republic of China

Tel/Fax +86-22-8333-6977

Email guoqijp@gmail.com
Purpose: We aimed to propose a serial mediational model to further analyze the relationship between poor physical performance, malnutrition, depression and cognitive impairment in Chinese community-dwelling older adults.

Patients and Methods: This study consisted of 1386 community-dwelling Chinese older adults aged 65 years and older in Shanghai, China. Mild cognitive impairment (MCI) was assessed by the Mini-Mental State Examination (MMSE) and Instrumental Activities Of Daily Living (IADL). Physical performance was assessed by short physical performance battery (SPPB). Malnutrition was defined with the Mini Nutritional Assessment (MNA). Depressive symptoms were evaluated by the 30-item Geriatric Depression Scale (GDS). Serial multiple mediator models were used.

Results: The mean age of the final analysis sample was $73.62 \pm 6.14$, and $57.6 \%(n=809)$ were females. The prevalence of MCI was $14.35 \%(n=199)$. Physical performance $(p<0.001)$, nutritional status $(\mathrm{p}=0.025)$, and depressive symptoms $(\mathrm{p}=0.002)$ were correlated with MCI. The serial mediational model revealed that MNA and GDS scores significantly mediated association of SPPB and MMSE scores ( $c^{\prime}=0.4728, \mathrm{p}<0.001$ ). Furthermore, depressive symptoms significantly mediated the association of physical performance and cognition $(p=0.0311)$, while malnutrition had no independent mediating effect between these two factors $(\mathrm{p}=0.794)$.

Conclusion: Our study examined the serial multiple mediation roles of nutritional status and depressive symptoms on the relationship between physical performance and cognitive function in community-dwelling Chinese older adults. Older adults who were in poor physical condition tend to have worse nutritional status, more severe depression, and poorer cognitive function.

Keywords: mild cognitive impairment, nutritional status, depressive symptoms, physical function

\section{Introduction}

The concept of mild cognitive impairment (MCI) is a state of cognitive function between that seen in normal aging and dementia. Its prevalence is estimated between $3 \%$ and $19 \%$ in older adults. ${ }^{1}$ MCI has a high risk of progression to dementia, but is considered to be a reversible process. ${ }^{1}$ Early recognition of high-risk older adults with MCI can better prevent the deterioration. Studies have recognized that the MCI is often affected by many factors including poor physical performance, which has a strong correlation with $\mathrm{MCI}^{2,3}$ In our previous work, we found that grip strength was 
associated with overall cognition, time orientation, recall, and language, while the Timed-up to go test and 4-m walking speed were associated with overall cognition and various cognitive domains, except recall. ${ }^{4}$ These results have not been fully supported by other reported studies. ${ }^{5,6}$ Thus, the role of confounders and the underlying mechanism remain incompletely defined, necessitating further studies.

Malnutrition and depression are also increasingly common health problems in the older adults, which are often considered as two harmful and potentially modifiable factors closely related to physical performance and MCI. Malnutrition is a chronic and frequently imperceptible process, and it is vital to identify it at an early stage. ${ }^{7}$ Studies have also found a link between nutritional status and cognition, ${ }^{8}$ cognitive decline may affect nutritional status and lead to decreased nutrient intake, and the resulting weight loss and malnutrition may worsen cognitive function. ${ }^{9}$ However, inconsistent results have also been reported that nutritional supplements did not improve cognitive function in older adults who were malnourished, ${ }^{10}$ and persons with MCI may not present major differences in nutritional status compared to healthy individuals. ${ }^{11}$ Therefore, the association between malnutrition and cognitive function needs to be further evaluated.

In addition, depression in older adults is a frequent psychiatric disorder, $10 \%$ to $15 \%$ have clinically significant depressive symptoms that affect their quality of life. ${ }^{12}$ Older adults with cognitive impairment were often affected by physical performance and psychological variables. ${ }^{13}$ Additionally, the effect between the change status of cognitive function and physical performance on the increase in depressive symptoms indicated a strong interaction between the three factors. ${ }^{14,15} \mathrm{~A}$ decline in cognition caused by low mood principally involved memory, executive function, and attention, often affected by physical activity. ${ }^{16}$ Furthermore, most studies suggested that there is a relationship between malnutrition and depression. ${ }^{17,18}$ But it is not clear whether depression is the cause or effect of malnutrition. ${ }^{19}$

To sum up, we hypothesize that MCI is associated with physical performance, malnutrition and depression. Although previous studies have explored their independent contributions, no studies have explored all these factors in combination, particularly in Chinese population. Therefore, we aim to propose a serial mediational model to further analyze the relationship between poor physical performance, malnutrition, depression, and cognitive impairment. This model may be used to elucidate relevant mechanisms, and to further identify and prevent MCI in Chinese community-dwelling older adults.

\section{Materials and Methods Participants}

Our research population included residents from Chongming, Shanghai, China, who had joined China's national free physical examination program between March 2019 and September 2020. Individuals were included as following criteria: ${ }^{4}$ (1) Older adults people $\geq 65$ years old; (2) Have lived in the community for at least one year; (3) Willing to participate in this study. Exclusion criteria: ${ }^{4}$ (1) General cognitive function was assessed using the Chinese version of the Matisse Dementia Rating Scale (CDRS), excluding participants who already had severe cognitive impairment and dementia or other neurodegenerative diseases; (2) those who could not take care of themselves and the instrumental activities of daily living scale were more than or equal to three items that could not be completed and could not walk independently; (3) people with hearing impairment, visual impairment and communication difficulties; (4) those who took sedatives in the last one or two months; (5) unable to perform the physical performance test. The methodological sessions were carried out in accordance with the approved guidelines and regulations. Those participants whose questionnaires $(\mathrm{n}=51)$ or physical performance tests $(\mathrm{n}=18)$ incomplete were excluded, the final analysis included 1386 participants. All participants were fully informed of the nature of research and signed an informed consent to participate in. This study was approved by ethics committee at Shanghai University of Medicine and Health Sciences, China.

\section{Assessment of Physical Performance}

The short physical performance battery (SPPB) has three timed components: a 4-meter usual-pace walk, three balance stances and five repeated chair stands. Component times are converted to component scores ranging from 0 to 4, with higher score corresponding to greater functional status. A score of 0 is given if the participant deems the task too unsafe to attempt or attempts the task but is unable to complete it. ${ }^{20}$ The three component scores are summed to yield a total score ranging from 0 to 12 . This is a widely used scale exploring the reduction of physical performance in older persons, particularly muscle strength, walking speed at usual pace, and balance. ${ }^{21,22}$ 
To avoid the measurements error, the assessments in our study were conducted by postgraduate students in the health field who had received special training for testing administered all tests as part of a standardized geriatric assessment. In addition, each item of functional assessments shall be the responsibility of one personnel to decrease the error caused by different performers who completed the data collection of all the participants involved.

\section{Assessment of Cognitive Function}

Cognitive function was assessed using the Chinese-version mini mental state examination (MMSE), which is a brief screening test for cognitive impairment. It consists of 11 items measuring orientation, registration, attention, calculation, recall, and language. The maximum total score is 30 , with higher scores indicating better status., ${ }^{4,22}$

\section{Definition of $\mathrm{MCl}$}

The definition was made according to the diagnostic criteria of Petersen. ${ }^{23}$ The MMSE and Instrumental Activities of Daily Living scale (IADL) to definition MCI, that is MMSE to determine cognitive impairment and IADL judged normal daily activity ability. The cut-off points used for cognitive impairment were as follow: $\leq 17$ for illiterate people, $\leq 20$ for people with primary school, and $\leq 24$ for people with middle school or higher. ${ }^{24}$ IADL scores $\geq 6$ indicates normal daily activity ability which includes 8 items, the score ranges from 0 to 8 points, with the higher scores indicating better daily activity ability. ${ }^{4,25}$

\section{Assessment of Depressive Symptom}

Depressive symptoms were screened by the Chinese version of Geriatric Depression Scale (GDS), which is a standardized self-reported questionnaire consisting of 30 dichotomous questions. Sum of the 30 items produced a score ranging from 0 to 30 , with greater values indicating increased severity. The higher the GDS score, the more severe the depressive symptoms, and substantial depressive symptoms were defined as a score 11 or above. ${ }^{15,26}$

\section{Assessment of Nutritional Status}

To evaluate the nutritional status, the Mini Nutritional Assessment (MNA) was used. MNA comprises 18 items and takes approximately $15 \mathrm{~min}$ to perform. Each item is weighted by giving different scoring points to each prespecified answer, with higher values indicating better nutrition. Participants who scored more than 23.5 were allocated to be well nourished, and participants with a score between 17 and 23.5 were at risk of malnutrition. Malnourished individuals scored lower than $17 .^{26}$

\section{Covariates}

All participants were invited to a face-to-face interview to answer a standardized questionnaire. Baseline measures of sociodemographic characteristics, health behaviors, condition of chronic diseases were considered as covariates. Demographic characteristics included age, gender, education level, registered residence and marital status. Health behaviors included smoking and drinking habits. Our study used the short form of the International Physical Activity Questionnaire (IPAQ) to assess physical activity. Doctors' diagnoses of chronic diseases considered were hypertension, dyslipidemia, diabetes, heart disease, stroke, osteoarthritis, gout, peptic ulcer, Parkinson and cancer. And we also used Bioimpedance analysis (In-Body 720; Biospace Co., Ltd., Seoul, Korea) to estimate appendicular skeletal mass (ASM).

\section{Statistical Analysis}

All statistical analysis was performed using IBM SPSS v26.0 software (SPSS Inc., Chicago, IL, USA). The significance level in the current research was set as 0.05 .

Baseline sociodemographic and health-related characteristics were compared between participants with and without MCI using an independent $t$-test for numeric variables and a chi-square test for categorical variables. Data with a normal distribution are expressed as the mean $\pm \mathrm{SD}$, and categorical variables are expressed as proportions.

Pearson correlation coefficients were used to measure the degree of association between two variables in the data. Statistical significance of the effects of mediation was examined through the application PROCESS developed by Hayes (2018), an approach based on an ordinary least square regression model and the bootstrap method. Bootstrap analyses were conducted according to the guidelines provided by Hayes, using the IBM SPSS PROCESS (written by Andrew F. Hayes) macro running Serial Multiple Mediation-Model 6. ${ }^{27}$ The statistical significance of the mediating variable was examined over 5000 bootstrap samples. This method generated an estimate of the indirect effect, including 95\% confidence intervals. When zero was not within the $95 \%$ confidence limits, one could conclude that the indirect effect was significantly different from zero at the $5 \%$ level 
$(p<0.05)$; thus, the effect of the independent variable on the dependent variable was mediated by the proposed mediating variable. The significance level in the current research was set as 0.05 . IBM SPSS v26.0 software (SPSS Inc., Chicago, IL, USA) was used to analyze the research data.

We built a model with SPPB score as the independent variables, MMSE score as dependent variables, the MNA score and GDS score variable as potential serial mediators used by PROCESS macro v3.4. In all analyses, adjustments for potential confounding variables. Including age, gender, BMI, IPAQ, ASM, farming, living alone, widowed, illiteracy, smoking, drinking, diabetes, hypertension, hyperlipidemia, heart disease, peptic ulcer and osteoarthritis were included.

\section{Ethics}

The study was approved by the Ethics Committee of Shanghai University of Medicine and Health Sciences and the methods were carried out in accordance with the principles of the Declaration of Helsinki.

\section{Results}

\section{Characteristics of the Participants}

Of the 1386 participants in the final analysis, 57.6\% $(n=809)$ were females. The mean age of the participants was $73.42 \pm 6.14$. More than half of the people were farmers, and most of the participants were in primary school or below. Moreover, the incidence of MCI in the study population was $14.35 \%(n=199)$, similar to our previous study result. ${ }^{4}$ Table 1 presents the characteristics according to the incidence of MCI. Participants with MCI were more likely to be females $(\mathrm{P}<0.001)$, widowed $(\mathrm{P}<0.001)$, farming $(\mathrm{P}<0.001)$, and living alone $(\mathrm{P}<0.001)$. Older adults with MCI had lower ASM $(\mathrm{P}=0.003)$ and less physical activity $(\mathrm{P}=0.012)$.

Over all the participants the mean total MMSE and SPPB scores were $24.62 \pm 4.72$ (range $0-30$ ) and 10.24 \pm 2.12 (range 0-12), respectively. The findings of the Pearson correlation tests are presented in Table 2, which indicated that a positive significant relationship was found between the physical performance and nutritional status. Moreover, both were also both correlated with depression and inversely associated with depressive symptoms. Broadly speaking, we found that there were positive significant relationships between physical performance and
Table I Baseline Subject Characteristics According to the Incidence of $\mathrm{MCl}$

\begin{tabular}{|c|c|c|c|}
\hline Characteristic & Normal $(n=1 \mid 87)$ & $\mathrm{MCl}(n=199)$ & $\mathbf{P}$ \\
\hline Age (y) & $73.01 \pm 5.842$ & $75.85 \pm 7.239$ & $<0.001$ \\
\hline Gender & & & $<0.001$ \\
\hline Men (\%) & 44.1 & 27.1 & \\
\hline Women (\%) & 55.9 & 72.9 & \\
\hline BMI (kg/h2) & $23.67 \pm 3.480$ & $23.61 \pm 3.289$ & 0.822 \\
\hline Widowed (\%) & 18.0 & 35.7 & $<0.001$ \\
\hline Living alone (\%) & 15.3 & 30.2 & $<0.001$ \\
\hline Farming (\%) & 51.3 & 67.3 & $<0.001$ \\
\hline Education & & & $<0.001$ \\
\hline Illiterate (\%) & 32.2 & 64.3 & \\
\hline $\begin{array}{l}\text { Primary school or } \\
\text { less (\%) }\end{array}$ & 35.9 & 12.1 & \\
\hline Junior high school (\%) & 24.9 & 17.6 & \\
\hline $\begin{array}{l}\text { Senior high } \\
\text { school (\%) }\end{array}$ & 5.1 & 5.0 & \\
\hline College (\%) & 0.8 & 0 & \\
\hline Smoking (\%) & 15.4 & 14.1 & 0.748 \\
\hline Drinking (\%) & 28.2 & 28.3 & 1.000 \\
\hline SPPB score & $10.37 \pm 2.042$ & $9.41 \pm 2.396$ & $<0.001$ \\
\hline Balance & $3.47 \pm 0.889$ & $3.23 \pm 0.998$ & 0.001 \\
\hline Walking speed & $3.78 \pm 0.595$ & $3.53 \pm 0.803$ & $<0.001$ \\
\hline Chair stands & $3.12 \pm 1.113$ & $2.65 \pm 1.238$ & $<0.001$ \\
\hline GDS score & $5.56 \pm 4.568$ & $6.70 \pm 5.418$ & 0.002 \\
\hline Depression & 11.5 & 17.7 & 0.014 \\
\hline MNA score & $24.62 \pm 2.471$ & $24.20 \pm 2.411$ & 0.025 \\
\hline Malnutrition & 2.4 & 2.5 & \\
\hline Risk of malnutrition & 34.3 & 43.7 & \\
\hline Well-nourished & 63.3 & 53.8 & \\
\hline ASM (kg) & $17.28 \pm 3.856$ & $16.36 \pm 3.313$ & 0.003 \\
\hline IPAQ (Met/week) & $7016.62 \pm 7002.34$ & $5700.33 \pm 5435.72$ & 0.012 \\
\hline \multicolumn{4}{|l|}{ Number of diseases } \\
\hline Diabetes (\%) & 16.3 & 16.6 & 0.913 \\
\hline Hypertension (\%) & 61.6 & 67.8 & 0.064 \\
\hline Hyperlipidemia (\%) & 12.6 & 9.0 & 0.185 \\
\hline
\end{tabular}

(Continued) 
Table I (Continued).

\begin{tabular}{|l|l|l|l|}
\hline Characteristic & Normal $(\mathbf{n}=1$ 1 87) & $\mathbf{M C l}(\mathbf{n = 1 9 9 )}$ & $\mathbf{P}$ \\
\hline Gout (\%) & 6.2 & 4.0 & 0.088 \\
\hline Stroke (\%) & 10.2 & 13.1 & 0.238 \\
\hline Heart disease (\%) & 33.5 & 35.2 & 0.331 \\
\hline Cancer (\%) & 3.9 & 3.0 & 0.336 \\
\hline Osteoarthritis (\%) & 16.0 & 20.6 & 0.096 \\
\hline Peptic ulcer (\%) & 14.4 & 10.6 & 0.149 \\
\hline Parkinson (\%) & 1.2 & 1.0 & 0.386 \\
\hline
\end{tabular}

Notes: Data are presented as means \pm SD or percentage (\%).

Abbreviations: BMI, body mass index; SPPB, Short Physical Performance Battery score; MNA, Mini Nutritional Assessment; GDS, Geriatric Depression Scale; ASM, Appendicular Skeletal Mass; IPAQ, International Physical Activity.

nutritional status with cognitive function, but a significant negative relationship between GDS score and cognitive function.

\section{Serial Multiple Mediational Analyses}

Figure 1 displays results for the tested models of the mediation roles of nutritional status and depression in the relationship between physical performance and cognitive function. The total indirect effect was 0.0398 ( $\mathrm{SE}=0.0165, \mathrm{p}<0.001)$; physical performance on cognitive function was at significant levels, indicating that physical performance is a protective factor for cognition which therefore suggests partial mediation.

As shown in Figure 1, the direct effect of physical performance on MNA $(\mathrm{B}=0.1731 \mathrm{SE}=0.0342, \mathrm{p}<0.001)$ was significant, the nutritional status of the participants with good physical activity is better. In addition, the SPPB score was negatively associated with GDS score $(B=0.3302$, $\mathrm{SE}=0.0748, \mathrm{p}<0.001)$. There was also a negative correlation between nutritional status and depressive symptoms $(B=$ $-0.4058, \mathrm{SE}=0.0625, \mathrm{p}<0.001)$, this relationship also between depression symptom and cognitive function $(B=-0.0519$, $\mathrm{SE}=0.0240, \mathrm{p}<0.05)$. However, our results also demonstrated that nutritional status alone did not mediate the relationships mentioned above $(\mathrm{B}=0.0930 \mathrm{SE}=0.0530, \mathrm{p}>0.05)$. Therefore, physical performance did have an impact on cognitive function through nutritional status and depression symptom serially. Based on this result, nutritional status and depression symptom can act as a series of mediators which was observed to mediate between physical performance and cognitive function.

The statistical significance of indirect effects in the tested model was examined through 5000 bootstrap samples. Estimates were taken within a 95\% confidence interval and bias-corrected. Accelerated results are presented in Table 3. As seen in Table 3, based on a bias-corrected and accelerated (BCa) confidence interval of $95 \%$, within a statistically significant comparison and outside the point estimate interval, the mediation role of depression alone was found to be stronger than the serial multiple mediation role of nutritional status and depression together.

\section{Discussion}

This study examined the serial multiple mediation roles of nutritional status and depressive symptoms on the relationship between physical performance and cognitive function. The results demonstrated that these roles and the separate mediation of single mediating variables had statistically significant relationships among these components of older adults.

\section{Correlation Between Physical Performance and Cognitive Function}

Previous studies have also reported the association between physical performance and cognitive decline. One systematic review indicated that gait appeared to be an indicator of health and function might predict adverse health-related cognitive outcomes. ${ }^{28}$ Veronese et $\mathrm{al}^{3}$ showed that slow walking speed precedes the onset of poor cognitive functions and poor SPPB scores were significantly associated with the onset of cognitive impairment in both males and females. Moreover, chair standing time predicted the onset of cognitive impairment in

Table 2 Means, Standard Deviations, and Correlations Among the Variables

\begin{tabular}{|l|l|l|l|l|l|l|}
\hline Variable & M & SD & I.SPPB & 2.MNA & 3.GDS & 4.MMSE \\
\hline I.SPPB & 10.24 & 2.122 & NA & & & \\
2.MNA & 24.56 & 2.466 & $0.238^{* *}$ & NA & & \\
3.GDS & 5.73 & 4.712 & $-0.235^{* *}$ & $-0.255^{* *}$ & NA & \\
4.MMSE & 24.62 & 4.723 & $0.421^{* *}$ & $0.180^{* *}$ & $-0.164^{* *}$ & NA \\
\hline
\end{tabular}

Note: $* * p<0.001$.

Abbreviations: M, means; SD, standard deviations; NA, not applicable; SPPB, Short Physical Performance Battery score; MNA, Mini Nutritional Assessment; GDS, Geriatric Depression Scale; MMSE, Mini Mental State Examination. 


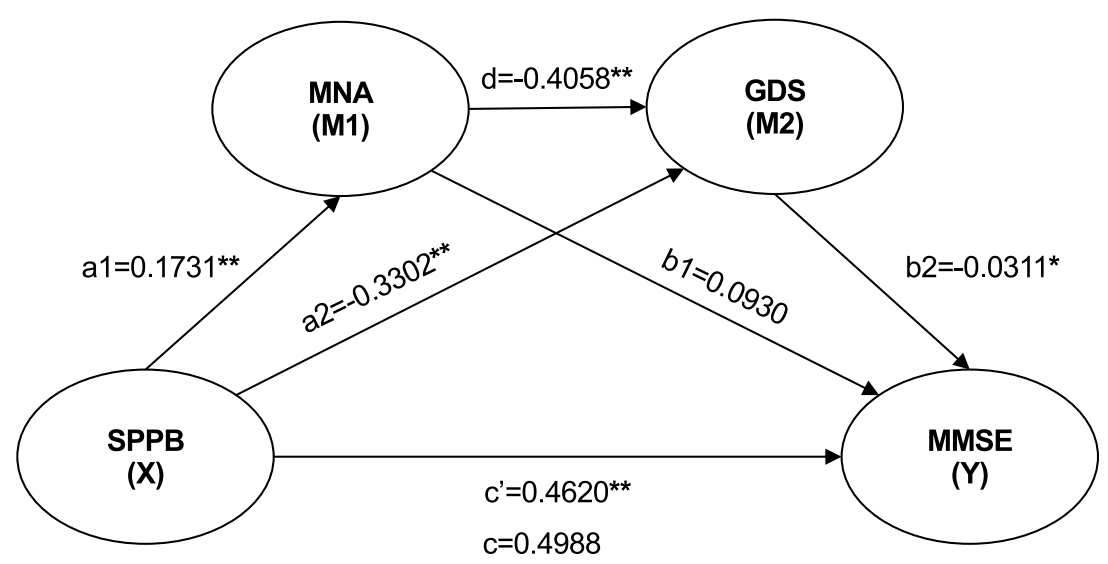

Figure I Nutritional status and depressive symptoms as mediators of physical performance and cognitive function.

Notes: ${ }^{* *} p<0.01 .{ }^{*} p<0.05$.

females. In our previous study, we found greater grip strength and faster walking speed were the protective factors for $\mathrm{MCI}^{4}$ Besides, Kuan et $\mathrm{al}^{2}$ suggested that regardless of musculoskeletal function, the early stage of MCI, will have adverse effects on balance function. Consistent with these results, our study further confirms the association between physical performance and cognition function $(\mathrm{P}<0.001$, 95\% CI: 0.3388-0.5852). But another study found that there was no significant correlation between upper muscle strength and cognitive function in Type 2 diabetes. ${ }^{5}$ The different results may be due to the specificity of the population, the results of our study need to be validated in other populations in the future.

Multiple potential explanations for the observed association between poor physical performance and cognitive impairment exist. One explanation may be that a neurodegenerative process or combinations of processes can lead to decreased motor and cognitive decline, but the impairment of these functions is detected at different times. Cognitive function may not deteriorate as rapidly as physical performance, especially in those with high educational attainment. Strategies for compensating cognitive loss might be more achievable than for physical impairment, but might be better at developing strategies to compensate for cognitive loss than for physical impairment, ${ }^{29}$ but the two factors might be closely related. Another explanation of this association could be that decline in physical performance contributes to physical inactivity, leading to decline in cognition. Physical activity increases the levels of several classes of growth factors, including brain-derived neurotrophic factor, ${ }^{30}$ insulin-like growth factor $1,{ }^{31}$ the peripheral serum BDNF level and reduced inflammation. ${ }^{32}$ Presently, these factors are considered to play a key role in regulating the effects of exercise on learning. This hypothesis could imply that poor physical performance is a potentially modifiable risk factor for cognitive decline in older adults.

\section{Nutritional Status and Mental Health as Mediators}

In a series of mediation model, we found that nutritional status and depression can act as mediators of physical performance and cognitive function. The decline of

Table 3 Specific Indirect Effects of Physical Performance Through Nutritional Status and Depression on Cognitive Function

\begin{tabular}{|l|l|l|l|l|}
\hline \multirow{2}{*}{ Effect } & Product of Coefficients & \multicolumn{3}{l|}{ Bootstrapping 95\% BCa Confidence Interval } \\
\cline { 2 - 5 } & Point Estimate & SE & Lower & Upper \\
\hline Total Indirect Effect & 0.0369 & 0.0150 & 0.0100 & 0.0696 \\
SPPB>MNA>MMSE & 0.0161 & 0.0103 & -0.0014 & 0.0393 \\
SPPB>GDS>MMSE & 0.0171 & 0.0092 & 0.0010 & 0.0365 \\
SPPB>MNA>GDS>MMSE & 0.0036 & 0.0023 & 0.0002 & 0.0090 \\
\hline
\end{tabular}

Notes: Model adjusted for age, gender, BMI, IPAQ, ASM, farming, living alone, widowed, Illiteracy, smoking, drinking, diabetes, hypertension, hyperlipidemia, heart disease, peptic ulcer, and osteoarthritis.

Abbreviations: SPPB, Short Physical Performance Battery score; MNA, Mini Nutritional Assessment; GDS, Geriatric Depression Scale; MMSE, Mini Mental State Examination. 
physical performance in the older adults' manifests malnutrition and depression, which are related to the decline of cognitive function $(\mathrm{B}=0.0036, \mathrm{SE}=0.0023,95 \% \mathrm{CI}$ : 0.0002-0.0090). This seems to be explained that physical performance decline, resulting in reduced activity, loss of appetite, resulting in bad emotions, and decline in cognition caused by low mood, often affected by physical activity. ${ }^{16}$ Moreover, malnutrition in the older adults was associated with MCI and depression symptomatology, independently of age, gender, educational level, BMI, and mid-arm and calf circumference. ${ }^{8}$ And cognition influences physical performance in older adults, also a significant interaction between these two factors could significantly accelerate decline of whole function in advanced age. ${ }^{33}$

The large sample size of study found that a tight association between malnutrition and depression was tight. In a study of older adults in Germany, the GDS score was the only factor that had a significant impact on nutritional status, ${ }^{18}$ and the risk of malnutrition had significantly higher GDS scores than patients with a normal nutritional status, after controlling for age, cognitive status, and functional ability. ${ }^{34}$ Our study also confirmed this relationship, showing a negative correlation between MNA scores and GDS scores was negative correlation. The mechanism between the two is still unclear, but it is believed that the lack of essential nutrients in the body of malnourished patients leads to a decrease in immune function, changes in neurohormones or neurotransmitters, and causes depression. ${ }^{35}$ Studies have found that the serum folic acid and vitamin B of patients with depression are lower than those of normal people. Older adults who lack folic acid, vitamin B12 and other antioxidants are more likely to have depressive symptoms. The deficiency of any metabolic material, such as protein, glucose and lipid, can lead to depression. ${ }^{36}$

When testing a single mediation model, the indirect effect of depression was significant for both physical performance and cognitive function $(\mathrm{B}=0.0171, \mathrm{SE}=0.0092$, 95\% CI: $0.0003-0.0412$ ). This result matched that of our recent study which found grip strength and walking speed are associated with MCI, and that depression might act as a mediator for both. ${ }^{15}$ When nutritional status was the only mediator, no association between physical performance and cognition was observed $(\mathrm{B}=0.0161, \mathrm{SE}=0.0103,95 \%$ CI:-0.0038-0.0407). This suggests that depression may be even more related to physical performance and cognitive function. Similarly, a previous study found that worsening cognitive function and deteriorating functional status synergistically influenced the development of depressive symptoms in older adults. ${ }^{37}$ This may be explained by the idea that older adults with worsening cognitive function may also have deteriorating functional status, and cognitive impairment may be aggravated, which in turn worsens daily living abilities. ${ }^{14}$ The vicious circle works as a sort of negative feedback loop, the risk attributed to worsening cognitive function or functional status is increased by the presence of the other. ${ }^{14}$ Another study found that patients receiving antidepressant treatment had worse physical and cognitive functions, which seems to indicate that taking antidepressants may affect cognitive and physical performance. $^{38}$

In addition, some studies have reported that nutritional problems, including appetite changes, weight loss, and sarcopenia, begin with MCI. ${ }^{39-41}$ One previous study reported that malnutrition can predict progression of MCI to dementia. ${ }^{23}$ However, separate work has shown that nutritional supplements do not improve cognitive function in older adults, ${ }^{10}$ possibly due to reduced digestive function and absorption ability. Although the detailed mechanisms of nutritional problems in MCI patients are not fully understood, nutritional problems appear to be important and modifiable factors that may affect the prognosis of MCI. In our study, nutritional status could not be used as a mediator alone. This result may be due to the small number of participants diagnosed with malnutrition in this study $(\mathrm{n}=33)$. Although many participants were at risk for malnutrition, the nutritional status of participants in this cohort was relatively good. Further research is needed to confirm this conclusion. At the same time, there is a strong correlation between reduced BMI and malnutrition, ${ }^{8}$ so BMI was controlled in the serial mediation model, and no association between $\mathrm{BMI}$ and $\mathrm{MCI}$ was found $(\mathrm{P}=0.822)$.

\section{Strengths and Limitations}

To the best of our knowledge, this study is the first time to incorporate physical performance, cognitive function, nutritional status, and depressive symptoms into a series of fully adjusted mediating models in Chinese communitydwelling older adults. This model seems to explain the mechanism behind the link between physical performance and cognitive function. Our study was cross-sectional, making it difficult to find the causal relationship between the various factors. Additionally, although our study controlled for several influencing confounding variables, we 
did not analyze the interaction between each variable. Finally, participants in this study were all relatively healthy older adults. Future work will need to increase the sample size, perform follow-up in this population to further verify the causal relationship, and validate these findings in other populations.

\section{Conclusion}

Our study further confirmed the association between physical performance and cognitive function in community-dwelling Chinese older adults. Nutritional status and mental health mediated between physical performance and cognitive function. Depressive symptoms also could play a mediating role in these two factors alone. These findings might help better early screening and management of MCI.

\section{Acknowledgments}

The authors thank all the members of the Department of Rehabilitation Medicine for their generous technical assistance and guidance. We also thank all the study participants for their kind participation and cooperation.

\section{Author Contributions}

All authors made substantial contributions to conception and design, acquisition of data, or analysis and interpretation of data; took part in drafting the article or revising it critically for important intellectual content; agreed to submit to the current journal; gave final approval of the version to be published; and agree to be accountable for all aspects of the work.

\section{Funding}

This work was supported by grant from Shanghai Sailing Program (20YF1418200), Scientific Research Foundation of SUMHS (SSF-21-03-005) and Wu Jieping medical foundation (320.6750.17073).

\section{Disclosure}

The authors report no conflicts of interest in this work.

\section{References}

1. Gauthier S, Reisberg B, Zaudig M, et al. Mild cognitive impairment. Lancet. 2006;367(9518):1262-1270. doi:10.1016/S0140-6736(06) 68542-5

2. Kuan Y, Huang L, Wang Y, et al. Balance and gait performance in older adults with early-stage cognitive impairment. Eur J Phys Rehabil Med. 2020. doi:10.23736/S1973-9087.20.06550-8
3. Veronese N, Stubbs B, Trevisan C, et al. What physical performance measures predict incident cognitive decline among intact older adults? A 4.4year follow up study. Exp Gerontol. 2016;81 (81):110-118. doi:10.1016/j.exger.2016.05.008

4. Liu Y, Ma W, Li M, et al. Relationship between physical performance and mild cognitive impairment in Chinese community-dwelling older adults. Clin Interv Aging. 2021;16:119-127. doi:10.2147/CIA. S288164

5. Low S, Ng T, Lim C, et al. Association between lower extremity skeletal muscle mass and impaired cognitive function in type 2 diabetes. Sci Rep. 2020;10(1):2956. doi:10.1038/s41598-020-59914-3

6. Donoghue O, Feeney J, O'Leary N, Kenny R. Baseline mobility is not associated with decline in cognitive function in healthy community-dwelling older adults: findings from The Irish Longitudinal Study on Ageing (TILDA). Am J Geriatr Psychiat. 2018;26(4):438-448. doi:10.1016/j.jagp.2017.11.006

7. Lahmann NA, Tannen A, Suhr R. Underweight and malnutrition in home care: a multicenter study. Clin Nutr. 2016;35(5):1140-1146. doi:10.1016/j.clnu.2015.09.008

8. Mantzorou M, Vadikolias K, Pavlidou E, et al. Nutritional status is associated with the degree of cognitive impairment and depressive symptoms in a Greek elderly population. Nutr Neurosci. 2020;23 (3):201-209. doi:10.1080/1028415X.2018.1486940

9. Landi F, Sieber C, Fielding R, Rolland Y, Guralnik J. Nutritional intervention in sarcopenia: report from the international conference on frailty and sarcopenia research task force. J Frail Aging. 2018;7 (4):247-252.

10. Baleztena J, Ruiz-Canela M, Sayon-Orea C, et al. Association between cognitive function and supplementation with omega-3 PUFAs and other nutrients in $\geq 75$ years old patients: a randomized multicenter study. PLoS One. 2018;13(3):e0193568. doi:10.1371/ journal.pone. 0193568

11. Cesari M, Azzolino D, Arosio B, Canevelli M. Nutritional interventions for early dementia. J Nutr Health Aging. 2021;25(5):688-691. doi:10.1007/s12603-021-1616-4

12. Kok R, Reynolds C. Management of depression in older adults: a review. JAMA. 2017;317(20):2114-2122. doi:10.1001/ jama.2017.5706

13. Kisvetrova H, Herzig R, Bretsnajdrova M, Tomanova J, Langova K, Skoloudik D. Predictors of quality of life and attitude to ageing in older adults with and without dementia. Aging Ment Health. 2021;25 (3):535-542. doi:10.1080/13607863.2019.1705758

14. Wang J, Zhu W, Li Y, Zhu W. Interaction between worsening cognitive function and deteriorating functional status on depressive symptoms among Chinese community-dwelling elders. Geriatr Gerontol Int. 2020;20(4):343-347. doi:10.1111/ggi.13887

15. Chen X, Han P, Yu X, et al. Relationships between sarcopenia, depressive symptoms, and mild cognitive impairment in Chinese community-dwelling older adults. $J$ Affect Disord. 2021;286:71-77. doi:10.1016/j.jad.2021.02.067

16. Mcclintock SM, Husain MM, Greer TL, Cullum CM. Association between depression severity and neurocognitive function in major depressive disorder: a review and synthesis. Neuropsychology. 2010;24(1):9-34. doi:10.1037/a0017336

17. Cabrera MAS, Mesas AE, Garcia ARL, De Andrade SM. Malnutrition and depression among community-dwelling elderly people. J Am Med Dir Assoc. 2007;8(9):582-584. doi:10.1016/j. jamda.2007.07.008

18. Smoliner C, Norman K, Wagner KH, Hartig W, Lochs H, Pirlich M. Malnutrition and depression in the institutionalised elderly. Br J Nutr. 2009;102(11):1663-1667. doi:10.1017/S0007114509990900

19. van Bokhorst-de van der Schueren M, Lonterman-Monasch S, de Vries O, Danner S, Kramer M, Muller M. Prevalence and determinants for malnutrition in geriatric outpatients. Clin Nutr. 2013;32 (6):1007-1011. doi:10.1016/j.clnu.2013.05.007 
20. Shea CA, Ward RE, Welch SA, Kiely DK, Goldstein R, Bean JF. Inability to perform the repeated chair stand task predicts fall-related injury in older primary care patients. Am J Phys Med Rehabil. 2018;97(6):426-432. doi:10.1097/PHM.0000000000000889

21. Lauretani F, Ticinesi A, Gionti L, et al. Short-Physical Performance Battery (SPPB) score is associated with falls in older outpatients. Aging Clin Exp Res. 2019;31(10):1435-1442. doi:10.1007/s40520018-1082-y

22. Fujita K, Nakashima H, Kako M, et al. Short physical performance battery discriminates clinical outcomes in hospitalized patients aged 75 years and over. Arch Gerontol Geriatr. 2020;90:104155. doi:10.1016/j.archger.2020.104155

23. Ye BS, Jang EY, Kim SY, et al. Unstable body mass index and progression to probable alzheimer's disease dementia in patients with amnestic mild cognitive impairment. $J$ Alzheimers Dis. 2016;49(2):483-491. doi:10.3233/JAD-150556

24. Zhang MY, Katzman R, Salmon D, Jin H, Levy P. The prevalence of dementia and Alzheimer's disease in Shanghai, China: impact of age, gender, and education. Ann Neurol. 2010;27(4):428-437. doi:10.1002/ana.410270412

25. Nagamatsu LS, Chan A, Davis JC, et al. Physical activity improves verbal and spatial memory in older adults with probable mild cognitive impairment: a 6-month randomized controlled trial. J Aging Res. 2013;2013:861893. doi:10.1155/2013/861893

26. Wang L, Wang X, Song P, et al. Combined depression and malnutrition as an effective predictor of first fall onset in a Chinese community-dwelling population: a 2-year prospective cohort study. Rejuvenation Res. 2020;23:498-507. doi:10.1089/rej.2019.2188

27. Hayes AF. Introduction to Mediation, Moderation, and Conditional Process Analysis: A Regression-Based Approach. Guilford publications; 2013.

28. Bortone I, Sardone R, Lampignano L, et al. How gait influences frailty models and health-related outcomes in clinical-based and population-based studies: a systematic review. $J$ Cachexia Sarcopenia Muscle. 2021;12:274-297. doi:10.1002/jcsm.12667

29. Bullain SS, Corrada MM, Shah BA, Mozaffar FH, Panzenboeck M, Kawas $\mathrm{CH}$. Poor physical performance and dementia in the oldest old: the 90+ study. JAMA Neurol. 2013;70(1):107-113. doi:10.1001/ jamaneurol.2013.583

30. Griffin ÉW, Mullally S, Foley C, Warmington SA, O’Mara SM, Kelly ÁM. Aerobic exercise improves hippocampal function and increases BDNF in the serum of young adult males. Physiol Behav. 2011.
31. Beine KH. Effect of resistance exercise on serum levels of growth factors in humans. Hormone Metabol Res. 2010;42(13).

32. Tsai CL, Pai MC, Ukropec J, Ukropcová B. Distinctive effects of aerobic and resistance exercise modes on neurocognitive and biochemical changes in individuals with mild cognitive impairment. Curr Alzheimer Res. 2019;16(4):316-332. doi:10.2174/ 1567205016666190228125429

33. Lauretani F, Maggio M, Ticinesi A, et al. Muscle weakness, cognitive impairment and their interaction on altered balance in elderly outpatients: results from the TRIP observational study. Clin Interv Aging. 2018;13:1437-1443. doi:10.2147/CIA.S165085

34. German L, Feldblum I, Bilenko N, Castel H, Harman-Boehm I, Shahar DR. Depressive symptoms and risk for malnutrition among hospitalized elderly people. J Nutr Health Aging. 2008.

35. Al-Rasheed R, Alrasheedi R, Johani RA, Alrashidi H, Alqadheb A. Malnutrition in elderly and its relation to depression. Int J Commun Med Public Health. 2018;5:2156. doi:10.18203/2394-6040. ijcmph20181974

36. Coppen A, Bolander-Gouaille C. Treatment of depression: time to consider folic acid and vitamin B12. J Psychopharmacol. 2005;19 (1):59-65. doi:10.1177/0269881105048899

37. Patnode CD, Perdue LA, Rossom RC, et al. Screening for cognitive impairment in older adults: updated evidence report and systematic review for the US Preventive Services Task Force. JAMA. 2020;323 (8):764-785.

38. Zainal N, Kalita P, Herr K. Cognitive dysfunction in Malaysian patients with major depressive disorder: a subgroup analysis of a multicountry, cross-sectional study. Asia Pacific Psychiatr. 2019;11(1):1-7. doi:10.1111/appy.12346

39. Shino S, Yutaka W, Hirohiko H, et al. Factors affecting the appetites of persons with Alzheimer's disease and mild cognitive impairment. Geriatr Gerontol Int. 2018;18(8):1236-1243. doi:10.1111/ggi.13455

40. Johnson DK, Wilkins CH, Morris JC. Accelerated weight loss may precede diagnosis in alzheimer disease. Arch Neurol. 2006;63 (9):1312-1317. doi:10.1001/archneur.63.9.1312

41. Sugimoto T, Ono R, Murata S, Saji N, Sakurai T. Prevalence and associated factors of sarcopenia in elderly subjects with amnestic mild cognitive impairment or Alzheimer disease. Curr Alzheimer Res. 2016;13(6):718-726. doi:10.2174/15672050136661602 11124828
Clinical Interventions in Aging

\section{Publish your work in this journal}

Clinical Interventions in Aging is an international, peer-reviewed journal focusing on evidence-based reports on the value or lack thereof of treatments intended to prevent or delay the onset of maladaptive correlates of aging in human beings. This journal is indexed on PubMed Central, MedLine, CAS, Scopus and the Elsevier
Bibliographic databases. The manuscript management system is completely online and includes a very quick and fair peer-review system, which is all easy to use. Visit http://www.dovepress.com/ testimonials.php to read real quotes from published authors. 